\title{
Updates on Baroreflex Activation Therapy and Vagus Nerve Stimulation for Treatment of Heart Failure With Reduced Ejection Fraction
}

\author{
Natasha Babar ${ }^{\mathrm{a}, \mathrm{b}, \mathrm{d}}$ (D), Dalia Giedrimiene $\mathrm{e}^{\mathrm{b}, \mathrm{c}}$
}

\begin{abstract}
In the last decade, neuromodulation via baroreflex activation therapy (BAT) and vagus nerve stimulation (VNS) has emerged as an innovative approach for the treatment of heart failure with reduced ejection fraction (HFrEF). A review of the literature was conducted to examine the latest efficacy and safety data on neuromodulation for the treatment of HFrEF. Two independent researchers searched the PubMed, clinicaltrials.org, and the Cochrane databases for the most recent data on BAT and VNS published between 2013 and 2019. A total of nine studies were identified. BAT and VNS therapy consistently improved subjective heart failure parameters including New York Heart Association class and Minnesota Living with Heart Failure Questionnaire. Improvements in objective cardiac parameters such as left ventricular ejection fraction (LVEF) were less consistently seen; however, where present, ranged from $+3 \%$ to $+6 \%$, in line with improvements seen after other guideline directed therapy such as left ventricular assist device (LVAD). Benefits of BAT showed a predilection for patients without cardiac resynchronization therapy (CRT) and efficacy of VNS therapy varied with device type. The clinical application of BAT and VNS was found to be limited due to low-powered data, inconsistencies in study design, short-term follow-up and lack of diversity in patient recruitment. Well-powered studies with consistent design, longer follow-up and diverse populations are warranted before BAT and VNS can be incorporated into heart failure guidelines and clinical practice.
\end{abstract}

Keywords: Neuromodulation; Heart failure with reduced ejection fraction; Baroreflex activation therapy; Vagal nerve stimulation; Advanced heart failure with reduced ejection fraction

Manuscript submitted October 14, 2021, accepted December 16, 2021

Published online January 10, 2022

a Department of Internal Medicine, North Shore University Hospital, Donald and Barbara Zucker School of Medicine at Hofstra/Northwell, Manhasset, NY, USA

${ }^{b}$ Frank H. Netter MD School of Medicine, North Haven, CT, USA

${ }^{\mathrm{c}}$ Clinical Research Center, Department of Cardiology, Hartford Hospital, Hartford, CT, USA

${ }^{\mathrm{d} C}$ Corresponding Author: Natasha Babar, Department of Internal Medicine, North Shore University Hospital, Donald and Barbara Zucker School of Medicine at Hofstra/Northwell, Manhasset, NY, USA.

Email: nbabar@northwell.edu

doi: https://doi.org/10.14740/cr1330
Introduction

Progression of heart failure with reduced ejection fraction (HFrEF) to an advanced state often necessitates the introduction of advanced therapies to relieve heart failure (HF) symptoms. Shortage of donor hearts, longer times on heart transplant waitlists, and transplant ineligibility combined with high mortality rates associated with HF (about $50 \%$ within 5 years of diagnosis [1]) have caused a move towards finding newer technologies to manage resistant HF symptoms.

In recent years, neuromodulation has been introduced as a novel approach for treatment of advanced HF. HFrEF involves hallmark anatomical and physiological changes including increased renin-angiotensin system activity, decreased vagal and increased sympathetic stimulation of the heart and body [2]. This results in tachyarrhythmias $[2,3]$, increased sympathetic stimulation of peripheral blood vessels [4], and diminished baroreceptor control over heart rate and peripheral resistance [4]. These hallmark changes act as compensatory mechanisms following the onset of HF but later contribute to the progression of the disease and have been linked to mortality in advanced HF patients [5,6]. Mechanistically, neuromodulation serves to counteract the physiological changes seen in advanced HF to improve functional capacity.

In this review, we highlight the most recent trends in the management of advanced heart failure, focusing on data from two new neuromodulatory device therapies, baroreflex activation therapy (BAT) and direct vagus nerve stimulation (VNS) between the years 2013 and 2019. Areas of progress, evaluation of evidence, remaining gaps in knowledge and recommendations for future study are presented herein.

\section{Methods}

A review of the literature was conducted using the PubMed, clinicaltrials.gov, and the Cochrane Library databases. The most recent studies reporting clinical outcomes published between 2013 and 2019 were included. Studies that reported statistical data with an $n \geq 30$ were preferred. Studies that did not report qualitative data and case reports were excluded. The search term "barostim" or "baroreflex activation therapy" AND "heart failure" was used for BAT while "vagus nerve stimulation" AND "heart failure" was used for VNS. Two in- 
dependent researchers were involved in literature review and data selection.

\section{Results}

\section{Literature review}

A total of nine studies representing the most recent data on neuromodulatory therapies for advanced heart failure were selected for further analysis (Table 1 [7-15]). Commonly reported patient baseline characteristics are summarized in Table 2 [7-15]. The mean age of study participants was 61.5 years. On average, $84 \%$ of study participants were male. The majority of study participants (85\%) had New York Heart Association (NYHA) class III HF with a mean left ventricular ejection fraction (LVEF) of 28\% across studies. Eight out of nine studies reported presence of implantable cardioverter defibrillator (ICD) therapy in their patients at baseline, with an average of $82 \%$ of study participants with ICDs.

\section{BAT}

Out of the advanced HF therapies chosen for review, BAT is the most recent with initial proof of concept studies dating back to 2014 [16]. BAT, also known as Barostim (Barostim ${ }^{\mathrm{TM}}$ neo $^{\mathrm{TM}}$, CVRx Inc., Minneapolis, MN, USA) has previously been shown to be safe and effective for the treatment of resistant hypertension [17], and is currently being explored for its ability to effectively counteract the autonomic imbalance present in HF $[7,16]$. BAT involves a battery operated, pacemaker-like pulse-generating device implanted in the chest wall with leads surgically placed in the carotid sinus. The leads provide continuous electrical stimulation at the carotid sinus baroreceptors to counteract the sympathetic overdrive that is seen in heart failure. The result is a centrally-mediated decline in sympathetic hyperactivity via vagal nerve response, leading to decreased heart rate and blood pressure [18].

Review of the literature showed BAT significantly decreased muscle sympathetic neuronal activity (MSNA), a surrogate for sympathetic activity, hospitalization rates, hospitalization days, NYHA class, LVEF, 6-minute hall walk distance (6MHWD) and Minnesota Living with Heart Failure Questionnaire score within a 6 - 43 month follow-up period (Table 1 [7-15]). In general, MSNA declined between -13.6 \pm 1.6 bursts $/ \mathrm{min}$ at 6 months [8] and $-19.6 \pm 4.4$ bursts $/ \mathrm{min}$ at 43 months of BAT [9]. HF hospitalization improved between $-6.28 \pm 2.7$ days at 6 months [10] and $-9.29 \pm 3.9$ days at 43 months [9]. LVEF improved +3.3 and +4.4 , and 6MHWD improved between $+69.7 \pm 24.4 \mathrm{~m}$ and $+106.8 \pm 58 \mathrm{~m}$ at 6 and 43 months $[8,9]$. An average improvement in at least a single NYHA class was seen at 6 or 43 months of BAT $[8,9]$. BAT was shown to be safe, with no device-related complications and major adverse neurological and cardiovascular events (MANCE)-free rate between 85\% and 100\% [10].

Importantly, the clinical benefits associated with BAT showed a predilection for patients without concurrent CRT [7] since significantly greater improvement in measures of functional capacity was noted in patients without CRT compared to those with CRT. One proposed explanation for this phenomenon as explained by BAT researchers is that by helping ventricles contract simultaneously, CRT may inhibit activation of the sympathetic baroreflex at the level of carotid baroreceptors, reducing the sympathovagal imbalance seen in advanced HF and thus limiting the effect of BAT [19]. Therefore, the suggested use for BAT is currently in NYHA III HF despite guideline-directed medical therapy (GDMT) only when not eligible for CRT [20].

\section{VNS}

Like BAT, autonomic regulation therapy (ART) via VNS is an emerging treatment for advanced HF in patients with reduced ejection fraction. VNS has previously been used as a safe and effective treatment for refractory epilepsy and depression. Similar to BAT, VNS serves to counteract the sympathetic overload seen in chronic heart failure by activation of a parasympathetic response [21]. To that end, an implantable neurostimulator activates the parasympathetic response via action of a pulse-delivering electrode placed at either cervical vagus nerve. A second, heart rate sensing electrode may be inserted directly into the right ventricle, which allows heart rate to be synchronized to pulse generation [21]. There are three different VNS devices that have been studied in clinical trials for HF: 1) the Cyberonics VNS System (Cyberonics, Houston Texas); 2) the Boston Scientific VNS system (St Paul, Minnesota, USA); and 3) the CardioFit System (BioControl Medical, Yehud, Israel). The devices differ by the degree of electrical stimulation applied and the presence of an intracardiac sensor lead, which is only found in the CardioFit system. In the studies under review, the Cyberonics system delivered $2.0 \mathrm{~mA}$ of electrical stimulation, the Boston Scientific $1.4 \mathrm{~mA}$ and the CardioFit system $4.1 \mathrm{~mA}$.

Review of the literature showed efficacy of VNS to vary with device type. The Cyberonics VNS device significantly increased LVEF ( $+4.5 \%, 95 \%$ confidence interval (CI): 2.4 6.6), 6MHWD (+56 m, 95\% CI: 37 - 75), and Minnesota Living with Heart Failure Questionnaire (MLHFQ) (-18 points, $95 \%$ CI: -20 to -13$)$ at 6 [11] and 12 months [12] with no statistical significant difference between findings at 6 and 12 months [12]. In comparison, the Boston Scientific VNS system showed increase in quality of life measures at 6 months including MLHFQ (-7.7 points, 95\% CI: -14.3 to -0.03$)$ and NYHA class ( +1 class in $62 \%$ of patients) while no significant improvement in cardiac parameters including left ventricular end systolic diameter (LVESD) or LVEF were found at either 6 [13] or 18 months [14]. The CardioFit device improved 6MHWD by $+28.2 \mathrm{~m}$ at 12 months $(\mathrm{P}<0.01)$ and NYHA class in $54.3 \%$ of patients; however no improvement in the primary end point of death from any cause or time to first event of worsening heart failure was found [15]. Overall, mortality in the VNS-treated patients ranged from 1.7\% [11] to $9.3 \%$ [15] and the most commonly reported adverse events were cough, dysphonia, implant site pain, and implant site infection [11]. 


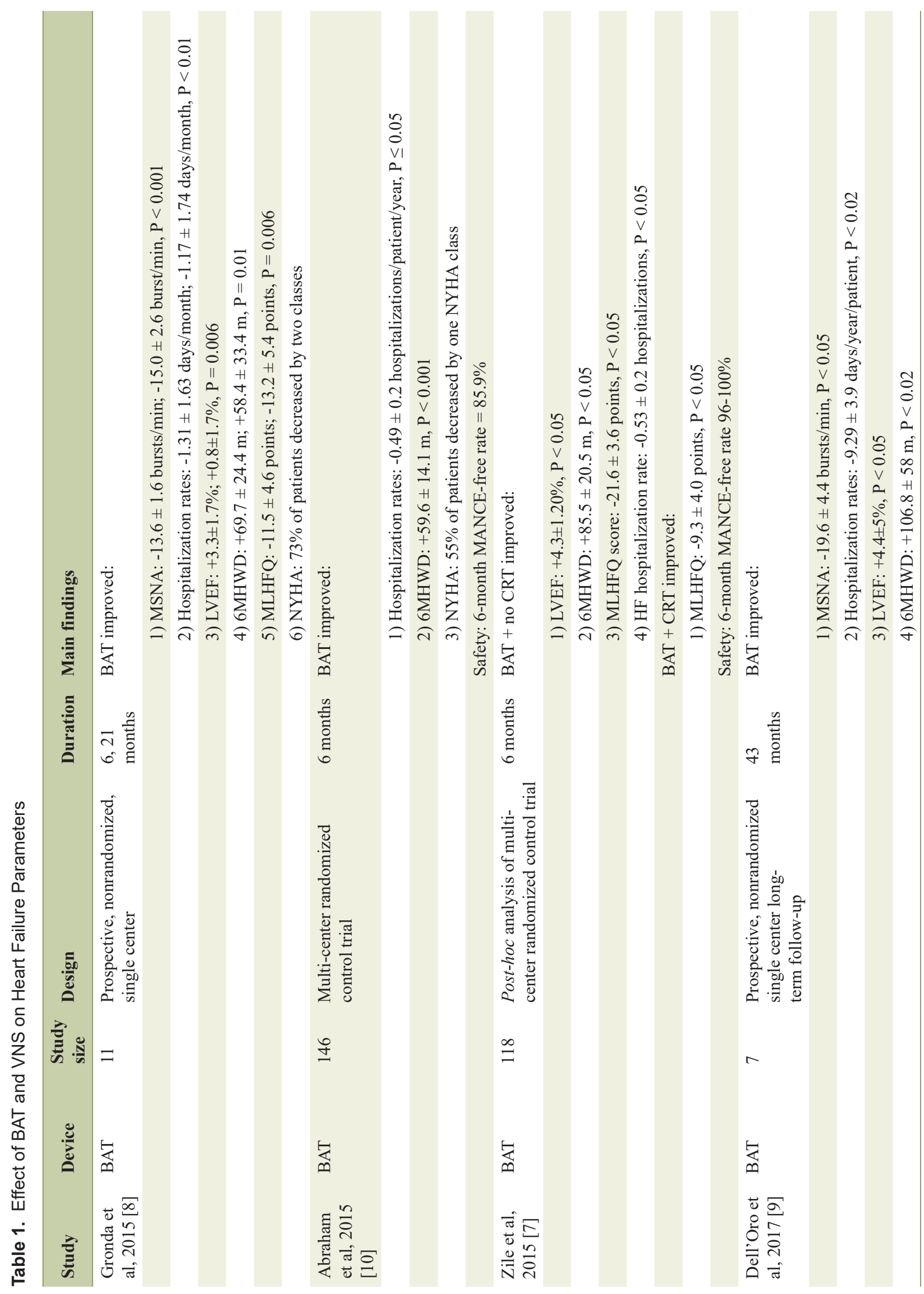




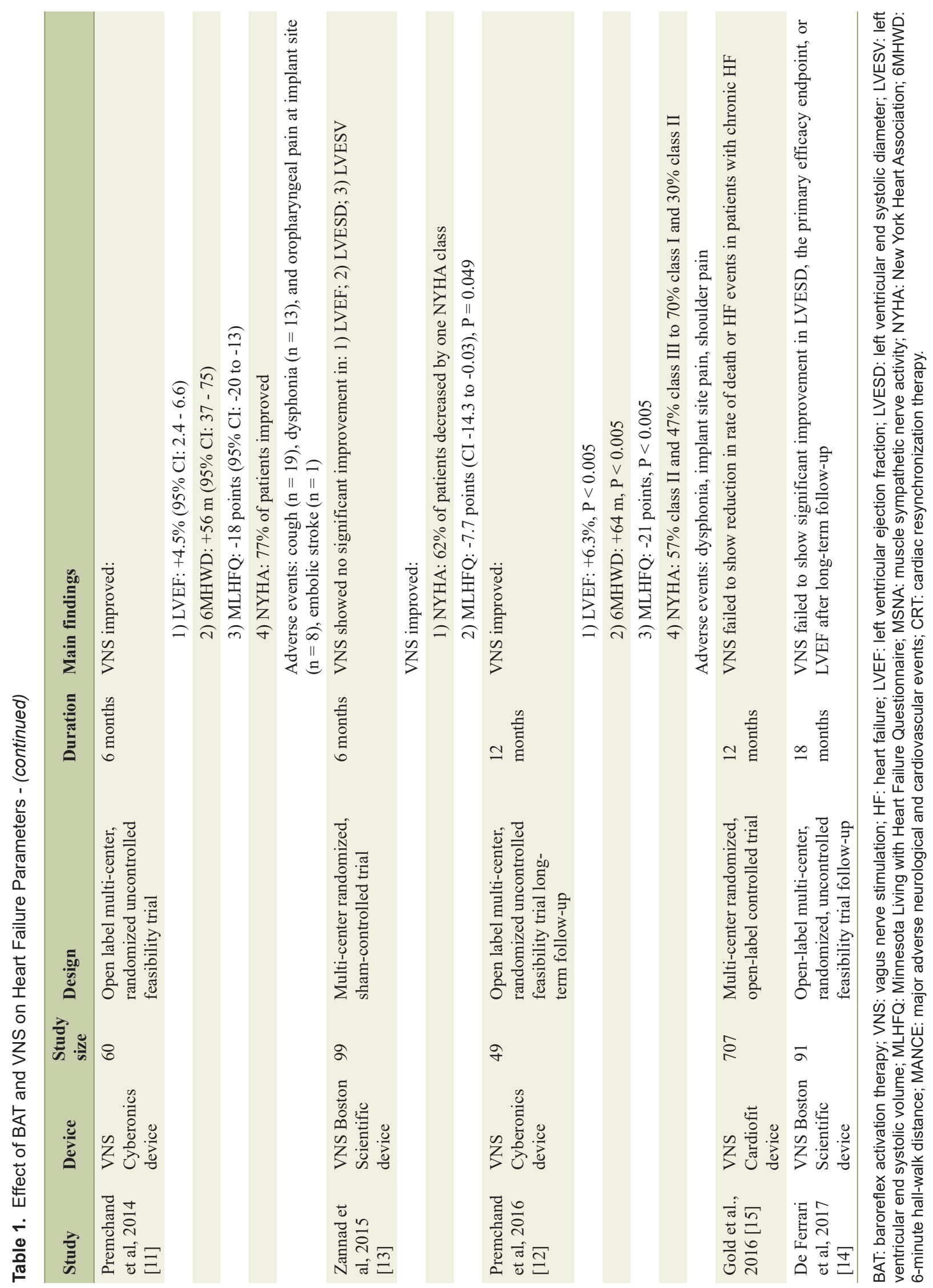


Table 2. Baseline Characteristics

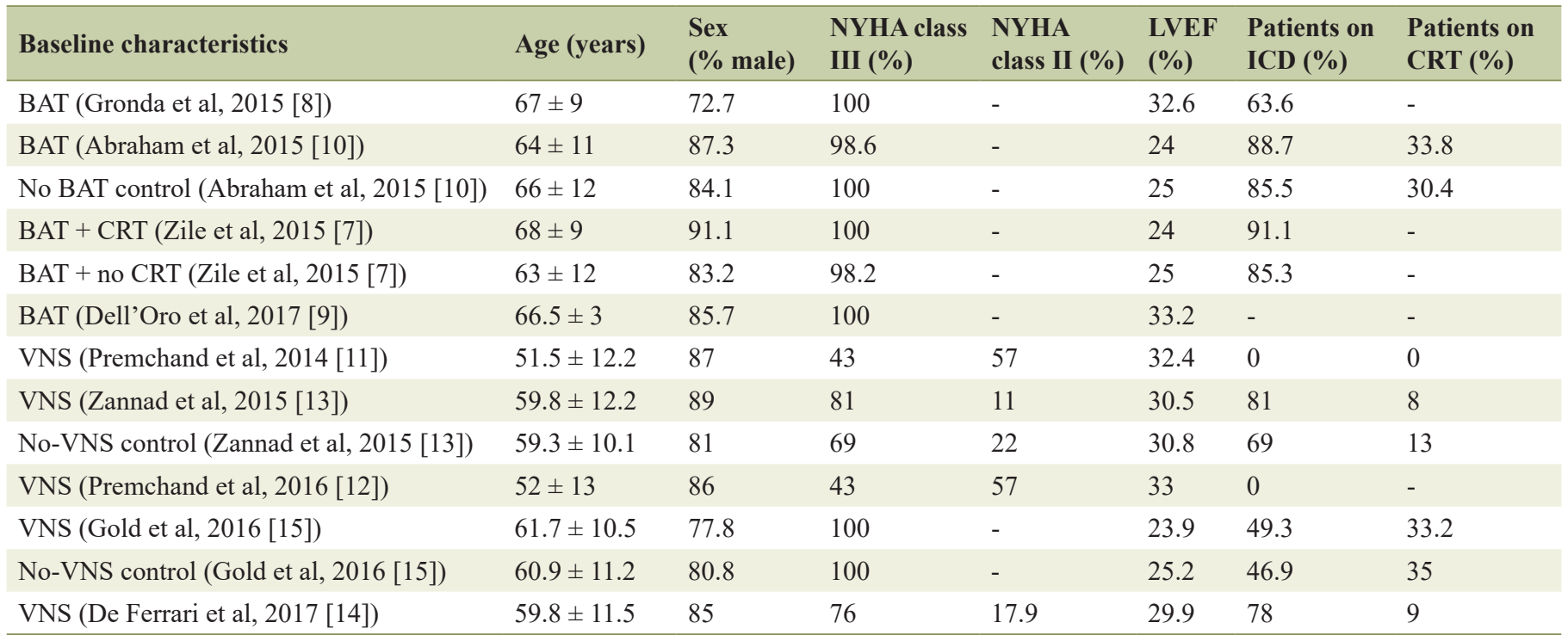

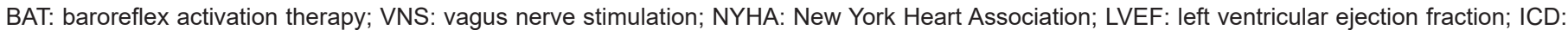
implantable cardioverter defibrillator; CRT: cardiac resynchronization therapy.

\section{Discussion}

In the last 10 years, neuromodulation via BAT and VNS has emerged as an innovative approach for the treatment of HFrEF. In this review, we summarized the recent efficacy data on BAT and VNS therapy. The total number of patients evaluated by our analysis were $n=157$ for BAT and $n=866$ for VNS. BAT and VNS showed improvement in subjective heart failure parameters such as NYHA class and MLHFQ almost consistently in all studies under investigation. Improvements in objective cardiac parameters such as LVEF and LVESD, however, were less consistently seen amongst both BAT and VNS studies. In studies where LVEF improved, the magnitude ranged from $+3 \%$ to $+6 \%$.

Our findings raise two important points. First, whether an improvement in LVEF by +3 to $+6 \%$ after neuromodulatory therapy translates to clinically significant improvement in cardiovascular status is considered. Interestingly, improvements in LVEF by $4-6 \%$ have been reported following LVAD therapy [22]. These findings suggest that the ability of BAT and VNS to improve cardiovascular parameters may be in line with more standard advanced therapies, such as LVAD. Second, it has been postulated that the improvement in subjective parameters such as NYHA class and MLHFQ seen after BAT and VNS can be explained by the placebo effect alone. Given the difficulty of blinding and having true negative controls with invasive device treatments, the degree to which the placebo effect has contributed to the improvements seen in subjective parameters may be difficult to ascertain. The placebo effect however does not by itself explain the near consistent improvement in 6MHWD seen after BAT and VNS therapy, ranging between $+30 \mathrm{~m}$ to $+100 \mathrm{~m}$. We postulate that by limiting sympathetic overdrive at the level of the heart and thus allowing more equal fractionation of sympathetic activity to other vital organs, neu- romodulation reduces vagally induced dyspnea at the level of lungs, thus improving functional capacity.

Despite these encouraging early findings, the data on BAT and VNS remains limited due to poor study design including low-powered direct or between-group comparison, absence of a comparison group, lack of long-term follow-up, and nonprimary design (Table 1 [7-15]). There was and still remains a general paucity of adequately powered, randomized studies on BAT and VNS therapy in HF patients. Keeping these limitations in mind, the use of BAT or VNS has yet to be included in official HFrEF guidelines due to lack of sufficient evidence in their favor [23].

A point on patient recruitment is worth highlighting. From close analysis of baseline characteristics reported in the studies under review, we found the majority of patients recruited for BAT and VNS therapy are on average about $80 \%$ male (Table 2 [7-15]), and where race was reported, 80-90\% Caucasian [7, $10,24]$. The lack of patient race and gender diversity in the articles chosen for our analysis is likely a reflection of study sites and recruitment, which are typically based in European populations. This, however, will be an important point to consider as future trials are planned elsewhere, including the USA, for the generalizability of clinical data and its ability to inform cardiology society guidelines.

\section{Limitations}

Though we provide a detailed account of the novel neuromodulatory therapies currently utilized for the treatment of advanced heart failure, our review carries some limitations. First, additional modes of neuromodulatory therapy beyond what is reported in this review exist for the treatment of advanced HF. One example is cardiac contract modulation where electrical 
stimulation is provided via pulse leads to the right ventricular septum during the ventricular refractory period with a goal of improving ejection fraction [25]. Our focus, however, was on the newest interventions with similar functionality which includes BAT and VNS. Our findings are additionally limited by the quality of studies under review, including an overall small patient size, particularly in the BAT group. The lack of more robust and higher powered studies likely is a reflection of the novelty of neuromodulation as a therapeutic approach for HFrEF. Finally, the inherent difficulty in blinding studies on device therapies serves as a limitation of this review. Use of an external sham control device would help overcome this limitation, as was seen in two out of the nine studies analyzed.

\section{Conclusions}

Analysis of recently published data shows successful progress in advanced therapies for HFrEF, including novel neuromodulatory therapies such as BAT and VNS. Despite early promising findings, the clinical application of BAT and VNS remains limited due to low-powered data, inconsistencies in study design, and lack of diversity in patient recruitment. Well-powered trials with consistent design, longer follow-up and recruitment of diverse populations are warranted before neuromodulation can be incorporated into official HF guidelines and thus into clinical practice.

\section{Acknowledgments}

None to declare.

\section{Financial Disclosure}

No fund, grant, or other support was received.

\section{Conflict of Interest}

The authors declare that they have no known competing financial interests or personal relationships that would influence the work reported in this paper.

\section{Author Contributions}

Both authors (Drs. Babar and Giedrimiene) contributed equally to the conceptualization, data gathering, and writing of this manuscript.

\section{Data Availability}

The authors declare that data supporting the findings of this study are available within the article.

\section{References}

1. Norton C, Georgiopoulou VV, Kalogeropoulos AP, Butler J. Epidemiology and cost of advanced heart failure. Prog Cardiovasc Dis. 2011;54(2):78-85.

2. Porter TR, Eckberg DL, Fritsch JM, Rea RF, Beightol LA, Schmedtje JF, Jr., Mohanty PK. Autonomic pathophysiology in heart failure patients. Sympathetic-cholinergic interrelations. J Clin Invest. 1990;85(5):1362-1371.

3. Georgakopoulos D, Little WC, Abraham WT, Weaver FA, Zile MR. Chronic baroreflex activation: a potential therapeutic approach to heart failure with preserved ejection fraction. J Card Fail. 2011;17(2):167-178.

4. Grassi G, Seravalle G, Cattaneo BM, Lanfranchi A, Vailati $\mathrm{S}$, Giannattasio C, Del Bo A, et al. Sympathetic activation and loss of reflex sympathetic control in mild congestive heart failure. Circulation. 1995;92(11):3206-3211.

5. Brunner-La Rocca HP, Esler MD, Jennings GL, Kaye DM. Effect of cardiac sympathetic nervous activity on mode of death in congestive heart failure. Eur Heart J. 2001;22(13):1136-1143.

6. Kaye DM, Lefkovits J, Jennings GL, Bergin P, Broughton A, Esler MD. Adverse consequences of high sympathetic nervous activity in the failing human heart. J Am Coll Cardiol. 1995;26(5):1257-1263.

7. Zile MR, Abraham WT, Weaver FA, Butter C, Ducharme A, Halbach M, Klug D, et al. Baroreflex activation therapy for the treatment of heart failure with a reduced ejection fraction: safety and efficacy in patients with and without cardiac resynchronization therapy. Eur J Heart Fail. 2015;17(10):1066-1074.

8. Gronda E, Seravalle G, Trevano FQ, Costantino G, Casini A, Alsheraei A, Lovett EG, et al. Long-term chronic baroreflex activation: persistent efficacy in patients with heart failure and reduced ejection fraction. J Hypertens. 2015;33(8):1704-1708.

9. Dell'Oro R, Gronda E, Seravalle G, Costantino G, Alberti L, Baronio B, Staine T, et al. Restoration of normal sympathetic neural function in heart failure following baroreflex activation therapy: final 43-month study report. J Hypertens. 2017;35(12):2532-2536.

10. Abraham WT, Zile MR, Weaver FA, Butter C, Ducharme A, Halbach M, Klug D, et al. Baroreflex activation therapy for the treatment of heart failure with a reduced ejection fraction. JACC Heart Fail. 2015;3(6):487-496.

11. Premchand RK, Sharma K, Mittal S, Monteiro R, Dixit S, Libbus I, DiCarlo LA, et al. Autonomic regulation therapy via left or right cervical vagus nerve stimulation in patients with chronic heart failure: results of the ANTHEMHF trial. J Card Fail. 2014;20(11):808-816.

12. Premchand RK, Sharma K, Mittal S, Monteiro R, Dixit S, Libbus I, DiCarlo LA, et al. Extended follow-up of patients with heart failure receiving autonomic regulation therapy in the ANTHEM-HF study. J Card Fail. 2016;22(8):639-642.

13. Zannad F, De Ferrari GM, Tuinenburg AE, Wright D, Brugada J, Butter C, Klein H, et al. Chronic vagal stimulation for the treatment of low ejection fraction heart fail- 
ure: results of the NEural Cardiac TherApy foR Heart Failure (NECTAR-HF) randomized controlled trial. Eur Heart J. 2015;36(7):425-433.

14. De Ferrari GM, Stolen C, Tuinenburg AE, Wright DJ, Brugada J, Butter C, Klein H, et al. Long-term vagal stimulation for heart failure: Eighteen month results from the NEural Cardiac TherApy foR Heart Failure (NECTARHF) trial. Int J Cardiol. 2017;244:229-234.

15. Gold MR, Van Veldhuisen DJ, Hauptman PJ, Borggrefe M, Kubo SH, Lieberman RA, Milasinovic G, et al. Vagus nerve stimulation for the treatment of heart failure: the INOVATE-HF trial. J Am Coll Cardiol. 2016;68(2):149158.

16. Gronda E, Seravalle G, Brambilla G, Costantino G, Casini A, Alsheraei A, Lovett EG, et al. Chronic baroreflex activation effects on sympathetic nerve traffic, baroreflex function, and cardiac haemodynamics in heart failure: a proof-of-concept study. Eur J Heart Fail. 2014;16(9):977983.

17. Alnima T, de Leeuw PW, Kroon AA. Baroreflex activation therapy for the treatment of drug-resistant hypertension: new developments. Cardiol Res Pract. 2012; 2012:587194.

18. Schmidli J, von Allmen RS, Mohaupt MG. Electrical carotid baroreceptor stimulation. Wien Med Wochenschr. 2014;164(23-24):508-514.

19. Gademan MG, van Bommel RJ, Ypenburg C, Haest JC, Schalij MJ, van der Wall EE, Bax JJ, et al. Biventricular pacing in chronic heart failure acutely facilitates the arterial baroreflex. Am J Physiol Heart Circ Physiol.
2008;295(2):H755-760

20. Gronda E, Francis D, Zannad F, Hamm C, Brugada J, Vanoli E. Baroreflex activation therapy: a new approach to the management of advanced heart failure with reduced ejection fraction. J Cardiovasc Med (Hagerstown). 2017;18(9):641-649.

21. Schwartz PJ, De Ferrari GM, Sanzo A, Landolina M, Rordorf R, Raineri C, Campana C, et al. Long term vagal stimulation in patients with advanced heart failure: first experience in man. Eur J Heart Fail. 2008;10(9):884-891.

22. Thiha S, Zaidi ARZ, Robert CA, Abbas MK, Malik BH. A Rising Hope of an artificial heart: left ventricular assisted device - outcome, convenience, and quality of life. Cureus. 2019;11(9):e5617.

23. Ponikowski P, Voors AA, Anker SD, Bueno H, Cleland JGF, Coats AJS, Falk V, et al. 2016 ESC Guidelines for the diagnosis and treatment of acute and chronic heart failure: The Task Force for the diagnosis and treatment of acute and chronic heart failure of the European Society of Cardiology (ESC)Developed with the special contribution of the Heart Failure Association (HFA) of the ESC. Eur Heart J. 2016;37(27):2129-2200.

24. Herscovici R, Kutyifa V, Barsheshet A, Solomon S, McNitt S, Polonsky B, Lee AY, et al. Early intervention and long-term outcome with cardiac resynchronization therapy in patients without a history of advanced heart failure symptoms. Eur J Heart Fail. 2015;17(9):964-970.

25. Duncker D, Veltmann C. Device therapy in heart failure with reduced ejection fraction-cardiac resynchronization therapy and more. Herz. 2018;43(5):415-422. 